\title{
The symmetry energy in nuclei and in nuclear matter
}

\author{
A.E.L. Dieperink ${ }^{1, a}$ and P. Van Isacker ${ }^{2}$ \\ 1 Kernfysisch Versneller Instituut, NL-9474AA Groningen, The Netherlands \\ 2 Grand Accélérateur National d'Ions Lourds, B.P. 55027, F-14076 Caen Cedex 5, France
}

Received: 15 January 2007

Published online: 27 April 2007 - (C) Società Italiana di Fisica / Springer-Verlag 2007

Communicated by G. Orlandini

\begin{abstract}
We discuss to what extent information on ground-state properties of finite nuclei (energies and radii) can be used to obtain constraints on the symmetry energy in nuclear matter and its dependence on the density. The starting point is a generalized Weizsäcker formula for ground-state energies. In particular, effects from the Wigner energy and shell structure on the symmetry energy are investigated. Strong correlations in the parameter space prevent a clear isolation of the surface contribution. Use of neutron skin information improves the situation. The result of the analysis appears consistent with a rather soft density dependence of the symmetry energy in nuclear matter.
\end{abstract}

PACS. 21.10.Dr Binding energies and masses $-26.60 .+\mathrm{c}$ Nuclear matter aspects of neutron stars $-21.10 . \mathrm{Ft}$ Charge distribution

\section{Introduction}

The nuclear symmetry energy is an important ingredient in the description of properties of proto-neutron stars. The equation of state, the proton fraction and the pressure are strongly affected by the density dependence of the symmetry energy in nuclear matter. Conventionally, the symmetry energy is expanded around the saturation density $\rho_{0}$ as $[1]$

$$
S(\rho)=a_{4}+p_{0}\left(\rho-\rho_{0}\right)+\Delta K\left(\rho-\rho_{0}\right)^{2} .
$$

Microscopic calculations are mostly based upon either models using realistic nucleon-nucleon interactions (based on Brueckner or variational techniques) or mean-field models using parameters fitted to data of finite nuclei. In practice, predictions for the symmetry energy vary substantially: e.g., $a_{4} \equiv S\left(\rho_{0}\right)=28-38 \mathrm{MeV}$, whereas predictions for the slope $p_{0}$ can vary by a factor three. A few observations are in order. First, general relativistic models - mean field as well as Dirac-Brueckner-HartreeFock $(\mathrm{BHF})$ - predict a substantially larger value for $p_{0}$ than non-relativistic ones. Secondly, in ab initio calculations there is also the uncertainty associated with a lack of a precise treatment of the effects from three-nucleon forces. Thirdly, even among the non-relativistic models (BHF and variational) there is no full agreement. A comparison of the results of the commonly used BHF approach

\footnotetext{
a e-mail: dieperink@kvi.nl
}

with more general "in-medium $T$ matrix" methods [2] (using the same nucleon-nucleon interaction) indicates that the BHF approach overestimates the symmetry energy.

In practice, only the empirical value of $a_{4} \sim 29 \mathrm{MeV}$ has been extracted with reasonable accuracy from finite nuclei by fitting ground-state energies using the Weizsäcker mass formula. Very little information (from data) on the slope, $p_{0}$, is available. From a recent analysis [3] of the excitation of the giant isoscalar monopole resonance in the $\mathrm{Sn}$ isotopes a negative curvature $\Delta K$ (asymmetric compressibility) was obtained, $-380<\Delta K<$ $-580 \mathrm{MeV}$.

Therefore the question arises whether one can obtain quantitative constraints from finite nuclei. Naturally, one may distinguish two regions, those containing information on sub-saturation densities $\left(\rho<\rho_{0}\right)$ and those on supranormal densities $\left(\rho>\rho_{0}\right)$. While the latter requires a (model-dependent) interpretation of results from heavyion reactions [4] (diffusion of neutron-proton asymmetry), the former region can be addressed by analyzing static properties of nuclei. This will be the subject of the present paper.

To make contact with finite systems, one uses the semiempirical mass formula $[5,6]$ which contains information on the average values of bulk and surface binding energies (for isospin symmetric systems) and (bulk) symmetry and Coulomb energies. In the past it has been realized that the symmetry and Coulomb energies not only have a bulk contribution but one from the surface as well. This leads to a generalized "liquid drop" description of finite 
nuclei (see Bohr and Mottelson [6], p. 621) and is akin to the treatment in nuclear collective models (e.g., describing properties of the giant dipole resonance) where it is equally essential to include a surface symmetry energy in addition to the bulk symmetry energy [7,8]. More recently, in connection with the semi-empirical mass formula, it has been stressed by Danielewicz [9] that in order to provide a consistent description of nuclei with neutron excess one must consider a surface symmetry term in addition to the bulk symmetry energy. The main purpose of this paper is to show that in considering a surface symmetry term several other corrections to the liquid-drop model (LDM) should be dealt with as well, in particular those due to shell structure and the effect of neutron-proton correlations (Wigner energy).

However, as in previous works, we find that volume and surface terms are strongly correlated and that the minimum in the deviation from observed masses is rather flat as a function of volume and surface energies along the correlation valley. As a consequence supplementary constraints on the surface symmetry energy are needed. The most direct one is the neutron-skin thickness as a function of nuclear asymmetry. Other potential sources of information on the symmetry energy in finite systems are related to properties of isovector giant resonances.

One of the main motivations of studies of this type is that it provides additional constraints on the nuclear equation of state. Once the nuclear surface symmetry energy is determined, it can be related to the symmetry energy $S(\rho)$ of nuclear matter at a sub-saturation density point either via a semi-infinite nuclear matter or via a Thomas-Fermi approximation.

This paper is organized as follows. In sect. 2 the surface symmetry energy in the extended liquid-drop model is presented and in sect. 3 the contributions from the Wigner energy and shell effects are discussed as well as the correlations in the parameter space. In sect. 4 the supplementary information on symmetry energies that can be obtained from data on neutron skins, isovector dipole giant resonances and isobaric analogue states is briefly presented. Constraints on the density dependence of the symmetry energy in infinite nuclear matter are discussed in sect. 5 . Section 6 contains a summary and concluding remarks.

\section{The extended liquid-drop model}

The conventional semi-empirical von Weizsäcker or liquiddrop mass (LDM) formula gives the binding energy of a nucleus as

$$
\begin{aligned}
B(N, Z)= & a_{\mathrm{v}} A-a_{\mathrm{s}} A^{2 / 3}-a_{\mathrm{s}}^{\prime} \frac{(N-Z)^{2}}{A} \\
& -a_{\mathrm{c}} \frac{Z(Z-1)}{A^{1 / 3}}+a_{\mathrm{p}} \frac{\Delta(N, Z)}{A^{1 / 2}},
\end{aligned}
$$

with $N$ and $Z$ the number of neutrons and protons, $A$ the total number of nucleons, $A=N+Z$. The terms on the right-hand side of (2) represent the bulk or volume, surface, symmetry, Coulomb and pairing energies, respectively, and $\Delta(N, Z)$ is a simple parametrization of pairing which is 1 for even-even, 0 for odd-mass and -1 for oddodd nuclei. The signs in eq. (2) are chosen such that all $a$ coefficients are positive.

It has been pointed out [9] that in eq. (2) volume and surface terms are not separated in a consistent way: one needs also to separate the volume and surface contributions to the symmetry energy. To accomplish this is not trivial. In a rigorous derivation one first introduces the concept of surface tension; the latter can then be decomposed into isospin symmetric and asymmetric contributions $[9,10]$. In practice the same result can be obtained in a more schematic way by decomposing the total particle asymmetry, $N-Z$, into volume (v) and surface (s) terms, $N-Z=N_{\mathrm{v}}-Z_{\mathrm{v}}+N_{\mathrm{s}}-Z_{\mathrm{s}}$, and requiring that the symmetry energy (quadratic in the asymmetry) scales with particle numbers as

$$
S_{\mathrm{v}} \frac{\left(N_{\mathrm{v}}-Z_{\mathrm{v}}\right)^{2}}{A}+S_{\mathrm{s}} \frac{\left(N_{\mathrm{s}}-Z_{\mathrm{s}}\right)^{2}}{A^{2 / 3}} .
$$

Minimization of eq. (3) under fixed $N-Z$ leads to a generalized formula for the binding energy of a nucleus in which the symmetry energy depends on two independent parameters, $S_{\mathrm{v}}$, the volume symmetry energy and the ratio $y_{\mathrm{s}} \equiv S_{\mathrm{v}} / S_{\mathrm{s}}$ with $S_{s}$ the surface symmetry energy:

$$
\begin{aligned}
B(N, Z)= & a_{\mathrm{v}} A-a_{\mathrm{s}} A^{2 / 3}-\frac{S_{\mathrm{v}}}{1+y_{\mathrm{s}} A^{-1 / 3}} \frac{(N-Z)^{2}}{A} \\
& -a_{\mathrm{c}} \frac{Z(Z-1)}{A^{1 / 3}}+a_{\mathrm{p}} \frac{\Delta(N, Z)}{A^{1 / 2}} .
\end{aligned}
$$

This expression for the nuclear binding energy forms the basis of the subsequent discussion. We will focus our attention in this contribution on two further corrections: those due to shell and deformation effects for which a simple parametrization shall be developed, and quantal corrections to the symmetry energy.

Before turning to the results obtained with refinements of the extended LDM (4), we point out that, with the use of a proportionality that exists between the neutron skin $\Delta R \equiv R_{\mathrm{n}}-R_{\mathrm{p}}$ and the nucleon surface asymmetry $N_{\mathrm{s}}-Z_{\mathrm{s}}$, the procedure leading to the result (4) also yields a direct relation between the skin $\Delta R$ and the symmetry energy parameters $\left(S_{\mathrm{v}}, S_{\mathrm{s}}\right)$ :

$$
\begin{aligned}
\frac{R_{\mathrm{n}}-R_{\mathrm{p}}}{R}= & \frac{A\left(N_{\mathrm{s}}-Z_{\mathrm{s}}\right)}{6 N Z}=\frac{A}{6 N Z} \\
& \times \frac{N-Z-a_{\mathrm{c}} Z A^{2 / 3}\left(12 S_{\mathrm{v}}\right)^{-1}}{1+y_{\mathrm{s}}^{-1} A^{1 / 3}},
\end{aligned}
$$

which is valid for the difference of sharp-sphere radii [9]. We note that in the absence of the Coulomb contribution the neutron skin depends on the ratio $y_{\mathrm{s}}$ only. However, except for very light nuclei, the Coulomb contribution cannot be neglected; for $N=Z$ nuclei it results in a proton skin and for $N>Z$ in a reduction of the neutron skin. For large $A$ and with $R \approx r_{0} A^{1 / 3}$, one finds from eq. (5) that $\Delta R / r_{0} \approx a_{\mathrm{c}}^{\prime}+b y_{\mathrm{s}}(N-Z) / A$, where $a_{\mathrm{c}}^{\prime}$ is a small negative function of $N$ and $Z$, representing Coulomb effects. 
In practice eq. (5) must be generalized for the case of rms radii. As pointed out by Danielewicz [9] this brings about an additional Coulomb correction (stemming from the polarization of the nuclear interior by the Coulomb force) to the right-hand side of eq. (5); the final result is [9]

$$
\begin{aligned}
& \frac{\left\langle r^{2}\right\rangle_{\mathrm{n}}^{1 / 2}-\left\langle r^{2}\right\rangle_{\mathrm{p}}^{1 / 2}}{\left\langle r^{2}\right\rangle^{1 / 2}}=\frac{A}{6 N Z} \\
& \times \frac{N-Z-a_{\mathrm{c}} Z A^{2 / 3}\left(10 / 3+y_{\mathrm{s}}^{-1} A^{1 / 3}\right)\left(28 S_{\mathrm{v}}\right)^{-1}}{1+y_{\mathrm{s}}^{-1} A^{1 / 3}},
\end{aligned}
$$

where a (very small) surface diffuseness contribution is neglected.

\section{Ground-state binding energies}

In fitting nuclear binding energies with the extended LDM formula (4), special care has to be taken with the treatment of $T=0$ pairing effects (or Wigner energy) — with an important impact on the symmetry energy - and of shell effects.

\subsection{Wigner energy}

Nuclei with $N=Z$ are in general more strongly bound as compared to the LDM formula; this effect can be incorporated by including an additional term (known as the Wigner energy) in mass formulas. The origin of the Wigner energy has been discussed by several groups, e.g., Satuła et al. [11-13], Zeldes [14], Neergåard [15] and Jänecke et al. $[16,17]$. The Wigner contribution to the binding energy is usually decomposed into two parts [18]

$$
B_{\mathrm{w}}(N, Z)=-W(A)|N-Z|-d(A) \delta_{N, Z} \pi_{\mathrm{np}},
$$

where $\pi_{\mathrm{np}}$ equals 1 for odd-odd nuclei and 0 otherwise. The origin of the Wigner energy (with $W(A)$ positive) can be understood microscopically as an effect from the overlap of neutron and proton wave functions which is maximal in $N=Z$ nuclei. The value of the parameter $W(A)$ can be determined in various ways, for example, from the double binding energy difference [19]

$$
\begin{aligned}
\delta V_{\mathrm{np}}(N, Z)= & \frac{1}{4}[B(N, Z)-B(N-2, Z) \\
& -B(N, Z-2)+B(N-2, Z-2)]
\end{aligned}
$$

valid for even-even nuclei, and differences thereof [11],

$$
W(A)=\frac{1}{2}\left[\delta V_{\mathrm{np}}(A / 2, A / 2-2)+\delta V_{\mathrm{np}}(A / 2+2, A / 2)\right] .
$$

To take into account the first term (linear in $T$ ) at the right-hand side of eq. (7), in several recent applications of the LDM the term $(N-Z)^{2}$ is replaced (in an ad hoc manner) by $4 T(T+r)$, where $T=\left|T_{z}\right|$ is the isospin of the nuclear ground state and $r$ is a parameter.
The form of the Wigner energy can be understood in more general terms from the supermultiplet theory [20]; the latter is based upon the assumption that nucleonnucleon forces are spin and isospin independent, and that as a result of the net attraction of the residual interactions the ground state has maximum spatial symmetry (or, equivalently, maximum $S U(4)$ anti-symmetry) consistent with the Pauli principle. As a result the correlation energy in the ground state is related to the expectation value of the quadratic $S U(4)$ Casimir operator in the following way [21]:

$$
\begin{aligned}
g(\lambda, \mu, \nu)= & (N-Z)^{2}+8|N-Z|+8 \delta_{N, Z} \pi_{\mathrm{np}} \\
& +6 \Delta^{\prime}(N, Z),
\end{aligned}
$$

where the labels $\lambda, \mu, \nu$ are functions of $N, Z$ and $\Delta^{\prime}(N, Z)$ is a pairing term which follows the somewhat unusual convention of being 0 in even-even, 1 in odd-mass and 2 in odd-odd nuclei. Note that the second and third terms exactly correspond to the Wigner energy (7) with the constraint $W=d$. Furthermore, it is seen that the first two terms in the expression (10) have the appearance of a symmetry energy $4 T(T+r)$ with $r=4$. (The correct $A$-dependence is lacking in eq. (10) since this information cannot be provided by supermultiplet theory.) If $S U(4)$ symmetry is broken entirely (as a result of the spin-orbit interaction) but isospin $S U(2)$ is conserved, an argument similar to that leading to eq. (10) gives a symmetry energy of the form $T(T+1)$, that is, $r=1$. One may thus expect that the coefficient $r$ lies somewhere between $r=1$ and $r=4$.

As a final remark in this section on the Wigner energy, we note that this effect is also at the basis of the frequently observed "isospin inversion" in odd-odd nuclei which should be taken into account in the fit to the nuclear masses.

\subsection{Shell effects}

It is well known that shell corrections to the LDM formula play an important role. In the literature many methods have been proposed to deal with shell effects, e.g., those developed by Möller and Nix [18]. Here we use a simple prescription which is closely related to the ideas used in the interacting boson approximation (IBA) model [22]. This model describes collective degrees of freedom in nuclei away from closed shells, and suggests that the relevant physics ingredient is the number of valence particles (neutrons and/or protons) with respect to the nearest closed shells (taken here to be $N, Z=20,28,50,82,126$ ) where particles beyond mid-shell are counted as holes. In our present work we add to the LDM expression a twoparameter term

$$
B_{\text {shell }}\left(N_{\mathrm{n}}, N_{\mathrm{p}}\right)=a_{1} F_{\max }+a_{2} F_{\max }^{2},
$$

where $F_{\max }=\left(N_{\mathrm{n}}+N_{\mathrm{p}}\right) / 2$ with $N_{i}$ the number of valence neutrons or protons, of particle or hole character. This is equivalent to counting bosons in the neutron-proton IBA model, where $F_{\max }$ is the maximum $F$ spin [23]. 

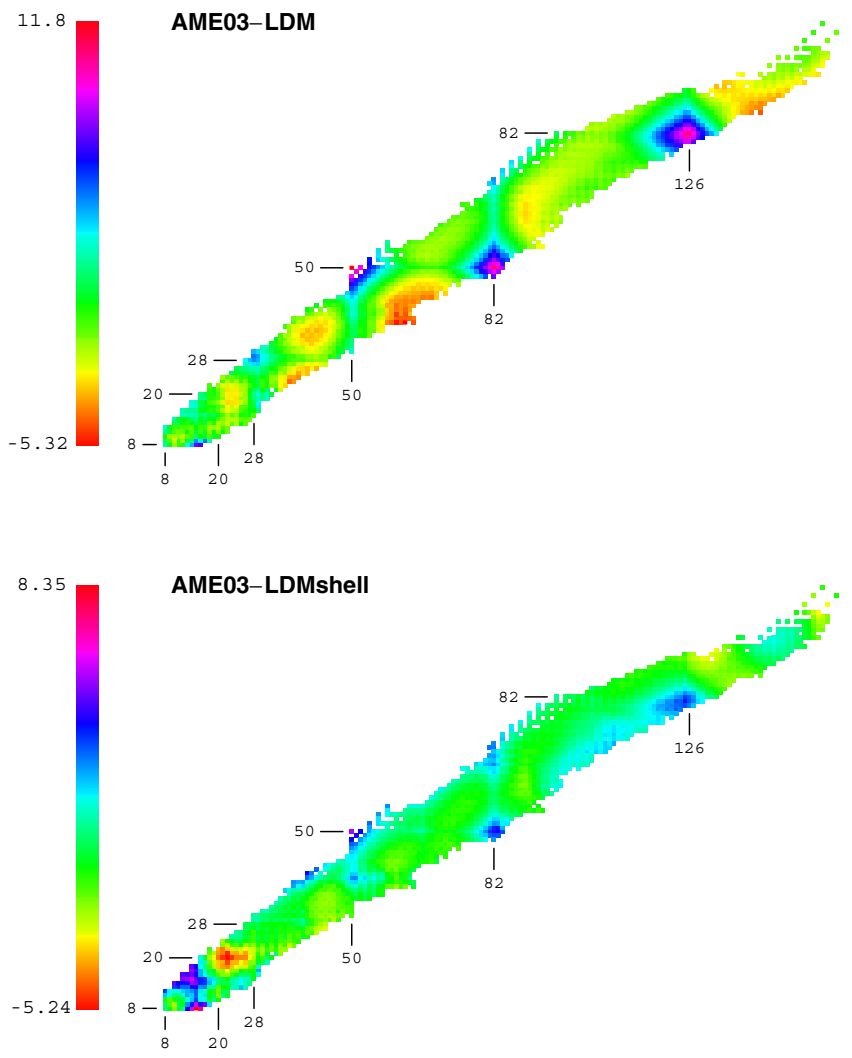

Fig. 1. (Color online) Differences between measured and calculated binding energies for nuclei with $N, Z \geq 8$. The binding energies are calculated with eq. (12) which includes surface symmetry and Wigner energies. The top figure is obtained without corrections due to shell structure while the bottom figure includes the two-parameter term (11).

\subsection{Results for fits to binding energies}

In all fits measured nuclear binding energies are taken from the 2003 atomic-mass evaluation [24]. All calculations are done with the extended LDM formula with a Wigner term,

$$
\begin{aligned}
B(N, Z)= & a_{\mathrm{v}} A-a_{\mathrm{s}} A^{2 / 3}-\frac{S_{\mathrm{v}}}{1+y_{\mathrm{s}} A^{-1 / 3}} \frac{4 T(T+r)}{A} \\
& -a_{\mathrm{c}} \frac{Z(Z-1)}{A^{1 / 3}}+a_{\mathrm{p}} \frac{\Delta(N, Z)}{A^{1 / 2}}
\end{aligned}
$$

to which the shell correction (11) is added in some cases. In fig. 1 we compare the results obtained with the extended LDM with $r=1$, with and without corrections due to shell structure. It is seen in the top figure (no shell corrections) that the large deviations around doubly magic nuclei have a diamond-like appearance and this suggests the use of a term linear in $F_{\max }$ which indeed provides an excellent parametrization of the shell corrections that are needed. Furthermore, the ellipse-like deviations in midshell regions suggest another term which is quadratic in $F_{\max }$. The use of these two simple corrections reduces the root-mean-square (rms) deviation from 2.47 to $1.40 \mathrm{MeV}$

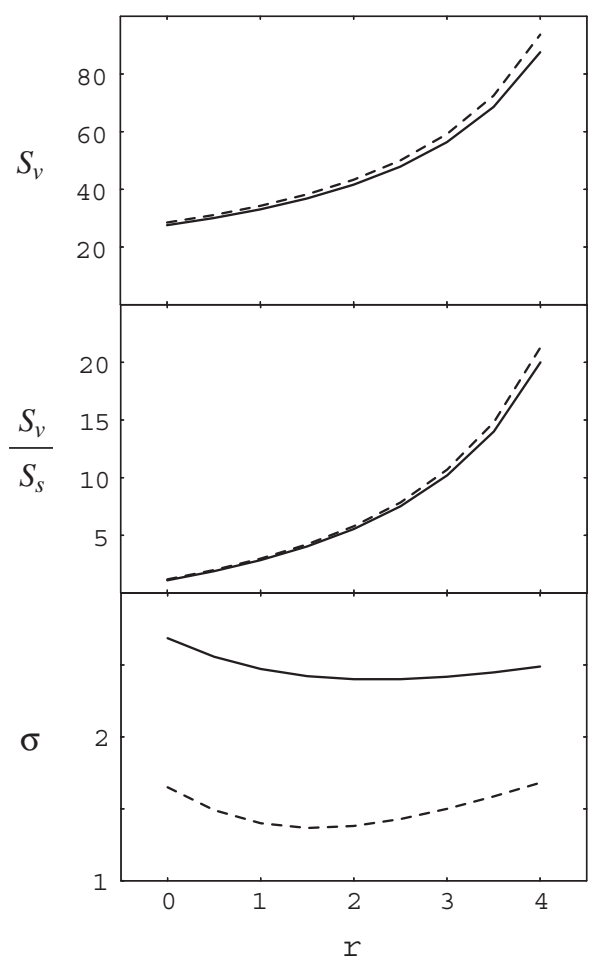

Fig. 2. The coefficient $S_{\mathrm{v}}$ (in $\mathrm{MeV}$ ), the ratio $y_{\mathrm{s}}=S_{\mathrm{v}} / S_{\mathrm{s}}$ and the rms deviation $\sigma$ (in $\mathrm{MeV}$ ) as a function of $r$, obtained with the LDM formula (4) without (full line) and with (dashed line) shell corrections.

while the values of the macroscopic coefficients remain stable. The shell-corrected plot (bottom of fig. 1) has much reduced deviations for the doubly magic nuclei and in the mid-shell regions of the heavier nuclei. A large fraction of the remaining rms deviation of $1.40 \mathrm{MeV}$ is due to nuclei lighter than ${ }^{56} \mathrm{Ni}$, where shell effects are large and cannot so easily be parametrized. An interesting feature of the shell-corrected plot is that it reveals additional or subshell effects: regions of larger deviations are seen around ${ }^{90} \mathrm{Zr}$ and ${ }^{146} \mathrm{Gd}$. These nuclei are known to have a doubly magic behavior associated with proton shell closures at $Z=40$ and $Z=64$, respectively. Since in the present fit shell closures are taken to be $N, Z=20,28,50,82,126$, such sub-shell effects are not included; a more refined algorithm for the choice of magic numbers is needed for this.

Figure 2 shows the coefficient $S_{\mathrm{v}}$, the ratio $y_{\mathrm{s}}=S_{\mathrm{v}} / S_{\mathrm{s}}$ and the rms deviation $\sigma$ as a function of $r$, obtained with the LDM formula (4) without and with shell corrections. Only the symmetry-energy coefficients $S_{\mathrm{v}}$ and $S_{\mathrm{s}}$ are sensitive to $r$ and all other coefficients in the LDM formula remain approximately constant. It is seen that the best fit values are quite sensitive to $r$ but the rms deviation is rather flat; on the other hand, the inclusion of shell effects reduces the rms deviation but hardly affects the best-fit values.

In table 1 the present results are compared with similar approaches of Danielewicz [9] and Steiner et al. [10] (model " $\mu_{\alpha}$ "). The smaller value of $y_{s}$ obtained with $r=0$ in the present work is not significant in view of the cor- 
Table 1. Values of $\sigma, S_{\mathrm{v}}$ and the ratio $y_{s}=S_{\mathrm{v}} / S_{\mathrm{s}}$.

\begin{tabular}{lccc}
\hline & $\sigma(\mathrm{MeV})$ & $S_{\mathrm{v}}(\mathrm{MeV})$ & $y_{\mathrm{s}}$ \\
\hline From binding energies [9] & 1.97 & 27 & 1.7 \\
From binding energies [10] & - & 27.3 & 1.7 \\
Present: Fit with LDM formula & 2.69 & 27.6 & 1.1 \\
$\quad+$ shell correction & 1.65 & 28.5 & 1.2 \\
$\quad$ + shell and Wigner corrections & 1.40 & 34.3 & 3.0 \\
\hline
\end{tabular}
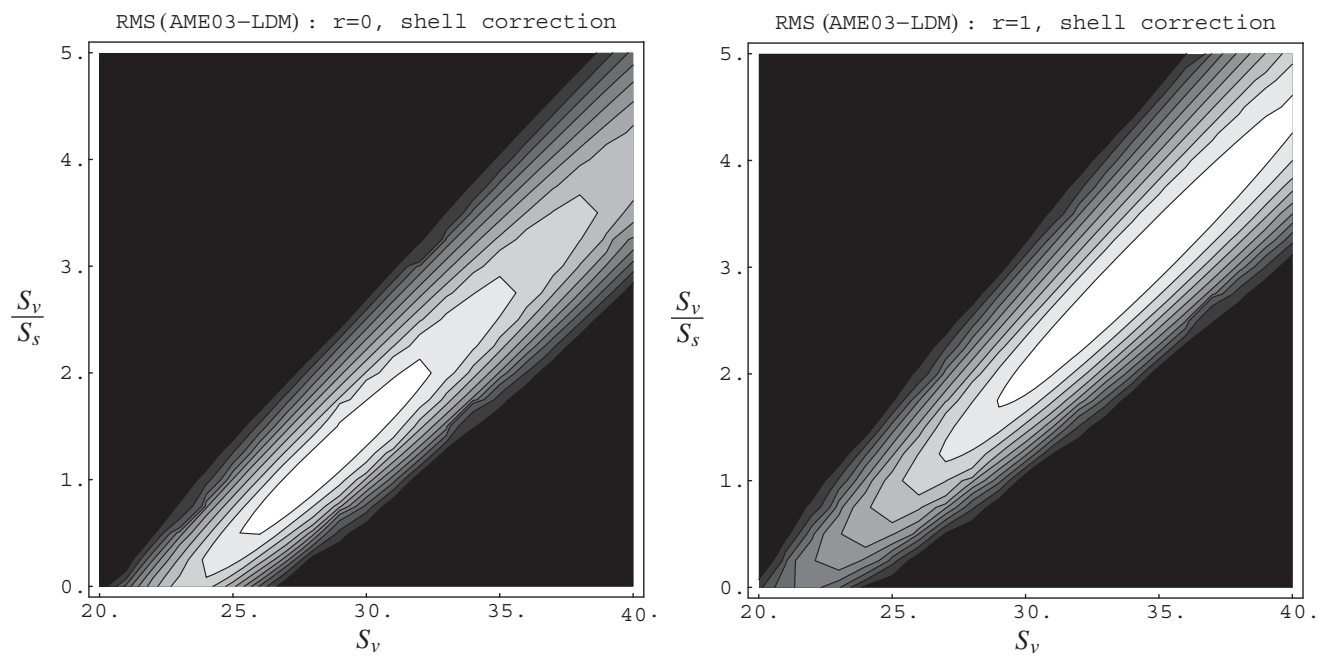

Fig. 3. Correlation plot of $y_{\mathrm{s}}=S_{\mathrm{v}} / S_{\mathrm{s}}$ versus $S_{\mathrm{v}}$ for $r=0$ (left) and $r=1$ (right). The white areas correspond to the combination of $S_{\mathrm{v}}$ and $y_{\mathrm{s}}$ giving rise to the lowest rms deviation, i.e., $1.65 \mathrm{MeV}$ for $r=0$ and $1.40 \mathrm{MeV}$ for $r=1$. Each contour interval corresponds to an increase of the rms deviation $\sigma$ by $100 \mathrm{keV}$.

relations between the parameters as discussed in the next subsection.

\subsection{Correlations between parameters}

It has been noted before by Danielewicz [9] and Steiner et al. [10] that in the fit to nuclear binding energies (assuming $r=0$ ) the parameters $S_{\mathrm{v}}$ and $S_{\mathrm{s}}$ are strongly correlated. In the correlation plot of $S_{\mathrm{v}}$ versus $y_{\mathrm{s}}=S_{\mathrm{v}} / S_{\mathrm{s}}$ one obtains for the rms deviation a narrow valley described by the linear relation $S_{\mathrm{v}}=a+b y_{\mathrm{s}}$. The actual values of $a$ and $b$ depend on details of the fitting procedure; e.g., $a=21.5$, 20.7 and $21.2 \mathrm{MeV}$, and $b=3.1,3.9$ and $6.1 \mathrm{MeV}$ in the model of Danielewicz [9] and in the models " $\mu_{\alpha}$ " and " $\mu_{n}$ " of Steiner et al. [10], respectively. In the present approach we obtain $a \approx 23 \mathrm{MeV}$ and $b \approx 4.2 \mathrm{MeV}$ (see fig. 3). This correlation can be understood qualitatively from the observation that one uses a two-parameter fitting function $S_{\mathrm{v}} /\left(1+y_{\mathrm{s}} A^{-1 / 3}\right)$, and that, in first approximation, for heavy nuclei $A^{-1 / 3}$ can be replaced by its average value (weighted with $\left.(N-Z)^{2} / A\right),\left\langle A^{-1 / 3}\right\rangle \approx 0.185$ for nuclei with $A>20$.

If one allows for $r>0$, the situation becomes even more complicated, as the correlation plot depends quite sensitively on the value of $r$. To see this, we write the generalized symmetry energy (third term at the right-hand side of eq. (12)) as

$$
\frac{S_{\mathrm{v}}}{1+y_{\mathrm{s}} A^{-1 / 3}} \frac{(N-Z)^{2}}{A}\left(1+\frac{2 r}{|N-Z|}\right) .
$$

The average value $\left\langle A^{-1 / 3} /|N-Z|\right\rangle \approx 0.203$ (weighted with $\left.(N-Z)^{2} / A\right)$ is about $10 \%$ larger than $\left\langle A^{-1 / 3}\right\rangle$. As a consequence, taking $r>0$ leads to a valley with a similar slope but with a somewhat smaller value (about 10\% for $r=1$ ) for the offset $a$. While the overall behavior of the correlation between $S_{\mathrm{v}}$ and the ratio $y_{\mathrm{s}}$ is rather insensitive to $r$, the best-fit values for $S_{\mathrm{v}}$ and $y_{\mathrm{s}}$ do vary substantially with $r: S_{\mathrm{v}}=28.5,34.3,38.2 \mathrm{MeV}$, and $y_{\mathrm{s}}=1.2$, $3.0,4.2$ with rms deviations of $1.65,1.40,1.37 \mathrm{MeV}$ for $r=0,1,1.5$, respectively. In particular, the best-fit value for $y_{\mathrm{s}}$ increases by a factor 2.5 by varying $r$ from 0 to 1 (see fig. 3) which reflects the existence of the strong correlation between $\langle|N-Z|\rangle$ and $\left\langle A^{1 / 3}\right\rangle$.

There is thus an obvious need to determine the value of $r$ independently. This issue has been studied to a certain extent by Jänecke et al. $[16,17]$ who treated the parameter $r$ as a mass-dependent shell effect. These authors observed a strong variation with mass region. In particular for nondiagonal regions (where neutrons and protons occupy different major shells, e.g., $50 \leq Z \leq 82$ and $82 \leq N \leq 126$ ) it was found that the value of $r$ is larger, $r \approx 2-4$ than in diagonal regions. However, in that analysis no surface symmetry energy term was considered; as a consequence 
the strong correlation between the parameters $r$ and $S_{\mathrm{s}}$ was ignored. We have tried to fit ground-state energies using eq. (4) with the parameter $r$ free to vary for different mass regions but could not establish a significant trend because of these correlations.

Admitting that the parameter $r$ cannot be reliably determined from ground-state energies, we have chosen $r=1$. This corresponds to the case of $S U(2)$ isospin symmetry, valid for a charge-independent nucleon-nucleon interaction, and is also used by Danielewicz [25].

\section{Supplementary information on the symmetry energy}

Since binding energies appear to constrain mainly a particular linear combination of the volume and surface symmetry-energy parameters, it appears desirable to employ supplementary information to obtain more reliable values of these parameters. To this end, one may use information from neutron skins or from collective isovector excitations.

\subsection{Neutron skin}

From eq. (5) it is clear that in the LDM there exists a direct relation between $\Delta R$ and $y_{\mathrm{s}}$ with a slight dependence on $S_{\mathrm{v}}$ coming from the Coulomb term $[9,10]$. We note that a similar correlation between (the derivative of) the symmetry energy in nuclear matter and $\Delta R=R_{\mathrm{n}}-R_{\mathrm{p}}$ for, e.g., ${ }^{208} \mathrm{~Pb}$ was observed by Brown [26] and by Furnstahl [27] in mean-field models.

One should realize that the extracted experimental information on $\Delta R$ is in general the result of a modeldependent analysis of nuclear reactions (elastic scattering of protons and neutrons, anti-protonic atoms, giantresonance excitations). Indeed, the values deduced for, e.g., ${ }^{208} \mathrm{~Pb}, \Delta R=0.10-0.20 \mathrm{fm}$, vary appreciably with the experimental approach and details of the analysis; also, in most cases the associated model uncertainties are difficult to quantify.

In order to minimize the model dependence, we have fitted data on radii obtained with one specific experimental tool only, namely from anti-protonic atoms, available for targets between ${ }^{40} \mathrm{Ca}$ and ${ }^{238} \mathrm{U}$ [28]. Therefore, we do not use information on unstable light nuclei, which we believe to be more model dependent. Since the dependence of $\Delta R$ on $S_{\mathrm{v}}$ is weak, we may adopt the value $S_{\mathrm{v}}=30 \mathrm{MeV}$ as it was obtained from ground-state energies, and determine the ratio $y_{\mathrm{s}}$ from the neutron skin data. The resulting fit obtained by using eq. (6) is shown in fig. 4 and yields a value of $y_{\mathrm{s}}=1.8$. It is seen that in the limited mass region considered the simple two-parameter fit $\Delta R=a+b(N-Z) / A=-0.03+0.90(N-Z) / A$ from Schmidt et al. [28] works equally well. That parametrization has, however, no obvious physical interpretation, the negative contribution from the $a$ coefficient becomes unphysical for light $N=Z$ nuclei and it does not properly describe the Coulomb term for heavy systems.

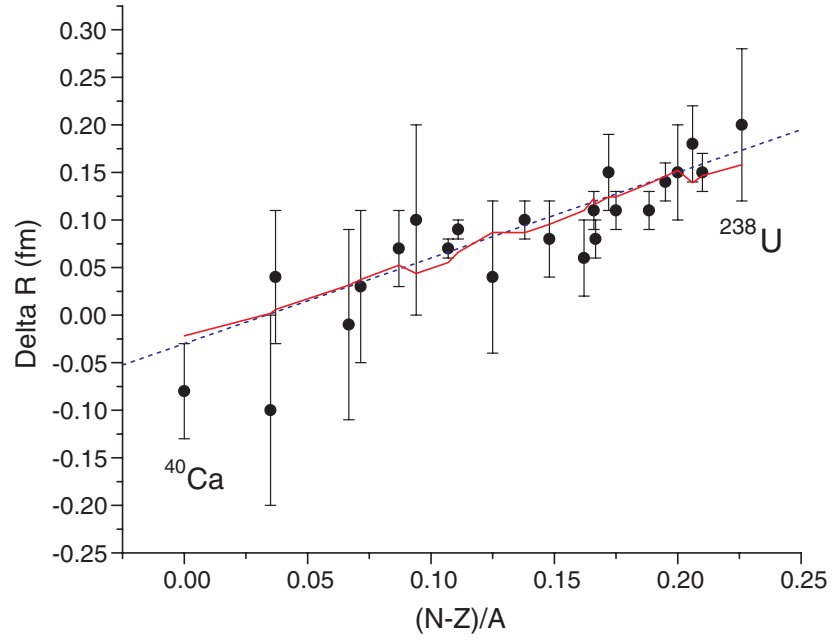

Fig. 4. The neutron skin data $\Delta R$ from anti-protonic atoms for nuclei between ${ }^{40} \mathrm{Ca}$ and ${ }^{238}$. The full line is obtained from the LDM expression (5) with a volume symmetry energy $S_{\mathrm{v}}=$ $30 \mathrm{MeV}$ and a volume-to-surface ratio $y_{\mathrm{s}}=1.8$. The dashed line is the fit $\Delta R=-0.03+0.90(N-Z) / A$ given by Schmidt et al. [28].

At this point one might wonder whether one should take into account shell corrections to radii, similar to the corrections to ground-state energies. In practice, one should expect two types of effects, namely collective ones related to the deformation, and single-particle effects from the filling of particular isolated sub-shells. The former could be included in a similar way as shell corrections to masses by adding terms linear in the number of valence nucleons. On the other hand, treating sub-shell effects (expected to be of importance near closed-shell nuclei like the filling of the $h_{11 / 2}$ neutron shell in the Sn isotopes) would require a more microscopic approach, which is beyond the scope of the present paper.

It is clear from fig. 4 that in practice in fitting $\Delta R$ over a larger mass region the symmetry energy is basically determined by the overall slope and that inclusion of shell effects even if they occur would not affect the result significantly.

\subsection{Isovector giant states}

Collective isovector excitations also contain information on the symmetry energy and/or the neutron skin. Since these modes have been studied recently in some detail, for completeness we briefly review the present status with the aim to see whether they can yield relevant constraints on the symmetry energy.

In a hydrodynamic approach the excitation energy of the isovector giant dipole resonance (IVGDR) depends on a combination of volume and surface symmetry energy and is given by [8]

$$
E_{\mathrm{GDR}}=\sqrt{\frac{6(1+K) S_{\mathrm{v}}}{m\left\langle r^{2}\right\rangle\left(1+5 y_{\mathrm{s}} A^{-1 / 3} / 3\right)}},
$$


where $K \sim 0.2$ is a dynamic enhancement factor. Using the values for the parameters $S_{\mathrm{v}}$ and $y_{\mathrm{s}}$ obtained above describes data for nuclei from ${ }^{40} \mathrm{Ca}$ to ${ }^{208} \mathrm{~Pb}$ reasonably well. The basic limitation in the quantitative application of this formula to determine $S_{\mathrm{v}}$ and $y_{\mathrm{s}}$ is the experimental uncertainty in $E_{\mathrm{GDR}}$.

Recently, it has been suggested that in heavy neutronrich nuclei the low-energy so-called pygmy dipole resonance (PDR), also contains relevant information on the surface symmetry energy. In a collective hydrodynamic approach the PDR has been regarded as an oscillation of the "excess neutron distribution" against the core [29]; these authors derived an expression for the fraction of the energy-weighted strength (relative to the GDR) which is proportional to the number of neutrons outside the core. This picture is qualitatively confirmed in a meanfield approach [30] where a linear correlation was found between the calculated fraction of the EWSR contained in the PDR and the neutron skin in the Sn isotopes below mass $A \approx 120$. In the light of the previous discussion one can easily understand this correlation if one interprets the excess neutron distribution as the surface asymmetry $N_{\mathrm{s}}-Z_{\mathrm{s}}$, quantity which determines both the surface symmetry energy and the neutron skin (see eq. (5)). However, in practice the validity of this correlation could be affected by sub-shell effects; e.g., in [30] it was observed that the $1 h_{11 / 2}$ neutron orbital contributes to the neutron skin but (with its large angular momentum) does not participate much in collective dipole transitions, and hence the filling of this orbital leads to a different correlation. Therefore more experimental work is needed to investigate the practical use of this information.

\subsection{Isobaric analogue states}

Recently, it was pointed out [25] that excitation energies of isobaric analogue states (IAS) in nuclei can also be used to determine the symmetry energy in a more direct way. The differences in excitation energies between states with isospin $T$ and $T^{\prime}$ in the same nucleus can be related to differences of masses in neighboring nuclei and a Coulomb correction, $\Delta_{T, T^{\prime}}\left(A, T_{z}\right)=M\left(A, T^{\prime}, T_{z}\right)-M\left(A, T, T_{z}\right)+$ $\Delta E_{\mathrm{c}}\left(T_{z}^{\prime}, T_{z}, A\right)-\left(T^{\prime}-T\right) \Delta M$, with $\Delta M$ the mass difference between neutron and proton. If one assumes charge independence of the nuclear Hamiltonian $H$, i.e., the matrix elements $\left\langle T, T_{z}|H| T^{\prime}, T_{z}\right\rangle$ are independent of $T_{z}$, the IAS energy can be expressed solely in terms of differences of symmetry and pairing energies $\Delta_{T, T^{\prime}}\left(A, T_{z}\right)=$ $E_{\text {sym }}\left(A, T^{\prime}\right)-E_{\text {sym }}(A, T)+E_{\text {pair }}\left(A, T^{\prime}\right)-E_{\text {pair }}(A, T)$. The advantage of using the IAS information compared to the LDM formula, eq. (12), is that by using differences of energies one can determine the symmetry energy in isolation of the isospin symmetric terms. Moreover, in special cases (odd $A$, and even $A$ if $\left|T-T^{\prime}\right|$ is even) the pairing contributions cancel. Hence, although the IAS does not contain new information compared to masses, the use of the IAS provides an additional tool to study the symmetry energy in an independent way. We have made an attempt to see whether in this way the parameter $r$ in the Wigner energy
Table 2. Values of $S_{\mathrm{v}}$ and the ratio $y_{s}=S_{\mathrm{v}} / S_{\mathrm{s}}$.

\begin{tabular}{lcc}
\hline & $S_{\mathrm{v}}(\mathrm{MeV})$ & $y_{\mathrm{s}}$ \\
\hline Masses + skins [9] & $27-31$ & $2.0-2.8$ \\
IASs + skins [25] & $30-32.5$ & $2.6-3.0$ \\
Present: Masses + skins & $31-33$ & $1.6-2.0$ \\
\hline
\end{tabular}

could be determined more reliably; however, that did not appear to be the case.

\subsection{Results}

In table 2 we compare the present results for the parameters $S_{\mathrm{v}}$ and $y_{\mathrm{s}}$ including the neutron skin information with those of Danielewicz $[9,25]$. Our approach seems to favor a smaller value for the ratio $y_{\mathrm{s}}$. It appears that this parameter is rather sensitive to details of the fitting procedure. For example, the fit to the IAS data in [25] involves a different sample of $(N, Z)$ nuclei than that of the experimental nuclear binding energies; also in our work we have used only information from data on neutron radii from anti-protonic atoms rather than information from radioactive beams.

\section{Asymmetric nuclear matter}

Clearly, the ratio $S_{\mathrm{v}} / S_{\mathrm{s}}$ is a measure for the density dependence of the symmetry energy in nuclear matter. In microscopic mean-field models one can at the same time compute isovector properties (like the neutron skin) in finite nuclei and the density dependence of symmetry energy in nuclear matter. In a macroscopic (liquid-drop) type of approach one may relate the ratio $S_{\mathrm{v}} / S_{\mathrm{s}}$ to $S\left(\rho_{0}\right) / S\left(\rho_{1}\right)$ at a selected sub-saturation density $\rho_{1}$ by integrating across the surface (using the Local Density Approximation) [9]:

$$
\frac{S_{\mathrm{v}}}{S_{\mathrm{s}}} \approx \frac{3}{R \rho_{0}} \int \rho(r)\left(\frac{S\left(\rho_{0}\right)}{S(\rho)}-1\right) \mathrm{d} r .
$$

This result can be derived from the minimization of the second-order (in the asymmetry) energy functional $E=$ $E_{0}+\int S(\rho) \rho\left(\rho_{\mathrm{a}} / \rho\right)^{2} \mathrm{~d} r$ under fixed particle numbers $A=$ $\int \rho \mathrm{d} r$ and $N-Z=\int \rho_{\mathrm{a}} \mathrm{d} r$ with $\rho_{\mathrm{a}} \equiv \rho_{\mathrm{n}}-\rho_{\mathrm{p}}$. Note that $S_{\mathrm{v}}=S\left(\rho_{0}\right)$; as a consequence, if the symmetry energy is independent of the density, $S(\rho)=S\left(\rho_{0}\right)=S_{\mathrm{v}}$, then $S_{\mathrm{s}}$ becomes infinite.

The application of eq. (15) becomes even simpler if one assumes a power parametrization for the density dependence of the symmetry energy, $S(\rho)=S_{\mathrm{v}} \times\left(\rho / \rho_{0}\right)^{\gamma}$. Using the value of $y_{\mathrm{s}}=1.8 \pm 0.3$ obtained from fitting neutron skin data, we find $\gamma=0.5 \pm 0.1$ although it is difficult to give a quantitative estimate of the error due to the Thomas-Fermi approximation. This result is consistent with $0.55<\gamma<0.79$ reported by Danielewicz [25]. It is also of interest to compare to the results from (relativistic) mean-field calculations. Recently, Piekarewicz [30] reported that the use of two sets of parameters, which both 
describe properties of finite nuclei, can lead to a drastically different density dependence of the symmetry energy: $S_{\mathrm{v}}=36.9(32.7) \mathrm{MeV}$ and $\gamma=0.98(0.64)$ for NL3 and FSUGold, respectively. There have been also attempts to constrain the value of $\gamma$ using heavy-ion reactions (isospin diffusion); it was found [31] that $0.7<\gamma<1.1$.

\section{Summary and conclusions}

Recent studies by Danielewicz [9] and Steiner et al. [10] have stressed the need for the inclusion of surface effects in the LDM formula, in particular in the symmetry energy. These authors showed that the bulk and surface symmetry energies are strongly correlated, rendering a reliable determination of these quantities from nuclear ground-state masses difficult. Data on neutron skins must be combined with those on masses to arrive at a separation of bulk and surface contributions.

In this paper we examined the influence of two further refinements of this extended LDM, namely nuclear shell structure and the Wigner energy. With the use of a simple parametrization of shell effects it was shown that, although a substantially smaller rms deviation is obtained with shell corrections than without, the correlation between the symmetry-energy parameters remains unchanged. So shell corrections do not substantially alter the observations made by Danielewicz [9] and Steiner et al. [10] as regards the correlation between the volume and surface symmetry-energy parameters. The conclusion is different, however, if a Wigner correction is added to the LDM formula. We have followed here the usual procedure of parametrizing the Wigner effect through $T(T+r)$ where $r$ is adjusted to mass data. Even if the decrease of the rms deviation due to this additional Wigner term is only about $300 \mathrm{keV}$, the effect of $r$ on the symmetryenergy parameters is spectacular. As a result, even more than without the Wigner correction, the separation of volume and surface symmetry-energy contributions can only be accomplished by use of data on neutron skins of nuclei.

The fact that the Wigner energy strongly influences the symmetry-energy parameters comes, with hindsight, as no surprise: a quantal calculation of the symmetry energy that takes account of the degeneracy structure of nuclei (either two-fold due to $S U(2)$ or four-fold due to $S U(4)$ symmetry) does lead in a natural way to a term linear in T. Symmetry and Wigner energies are thus closely related and one expects a strong correlation between them.

We have not been able to devise a procedure for a reliable determination of the Wigner parameter $r$ from nuclear ground-state binding energies, possibly because $T(T+r)$ is an inadequate parametrization of the Wigner effect. Nevertheless, in spite of this difficulty, one clear qualitative conclusion can be drawn from the present study: the Wigner effect implies a non-zero $r$ value which unavoidably leads to a larger value of $S_{\mathrm{v}}$, the symmetry energy of nuclear matter at the saturation point.

\section{References}

1. J.M. Lattimer, M. Prakash, Astrophys. J. 550, 426 (2001).

2. A.E.L. Dieperink, Y. Dewulf, D. Van Neck, M. Waroquier, V. Rodin, Phys. Rev. C 68, 064089 (2003).

3. M. Fujiwara, private communication.

4. P. Danielewicz, nucl-th/0512009.

5. C.F. von Weizsäcker, Z. Phys. 96, 431 (1935).

6. A. Bohr, B.R. Mottelson, Nuclear Structure II. Nuclear Deformations (Benjamin, New York, 1975).

7. E. Lipparini, S. Stringari, Phys. Lett. B 99, 183 (1981).

8. E. Lipparini, S. Stringari, Phys. Rep. 175, 103 (1989).

9. P. Danielewicz, Nucl. Phys. A 727, 233 (2003).

10. A.W. Steiner, M. Prakash, J.M. Lattimer, P.J. Ellis, Phys. Rep. 411, 325 (2005).

11. W. Satuła, D.J. Dean, J. Gary, S. Mizutori, W. Nazarewicz, Phys. Lett. B 407, 103 (1997).

12. W. Satuła, R. Wyss, Phys. Rev. Lett. 86, 4488 (2001); 87, 052504 (2001).

13. W. Satuła, R. Wyss, Phys. Lett. B 572, 152 (2003).

14. N. Zeldes, Phys. Lett. B 429, 20 (1998).

15. K. Neergåard, Phys. Lett. B 537, 287 (2002).

16. J. Jänecke, T.W. O'Donnell, V.I. Goldanskii, Nucl. Phys. A 728, 23 (2003).

17. J. Jänecke, T.W. O’Donnell, Phys. Lett. B 605, 87 (2005).

18. P. Möller, R. Nix, Nucl. Phys. A 536, 20 (1992).

19. J.-Y. Zhang, R.F. Casten, D.S. Brenner, Phys. Lett. B 227, 1 (1989).

20. E.P. Wigner, Phys. Rev. 51, 106; 947 (1937).

21. D.D. Warner, M. Bentley, P. Van Isacker, Phys. Nature 2, 311 (2006).

22. F. Iachello, A. Arima, The Interacting Boson Model (Cambridge University Press, Cambridge, 1987).

23. A. Arima, T. Otsuka, F. Iachello, I. Talmi, Phys. Lett. B 66, 205 (1977).

24. G. Audi, A.H. Wapstra, C. Thibault, Nucl. Phys. A 729, 337 (2003).

25. P. Danielewicz, nucl-th/0411115.

26. B.A. Brown, Phys. Rev. Lett. 85, 5296 (2000).

27. R. Furnstahl, Nucl. Phys. A 706, 85 (2002).

28. R. Schmidt et al., Phys. Rev. C 67, 044308 (2003); J. Jastrzebski, private communication.

29. Y. Suzuki et al., Prog. Theor. Phys. 83, 18 (1990).

30. J. Piekarewicz, Phys. Rev. C 73, 044325 (2006).

31. B.-A. Li, A.W. Steiner, Phys. Lett. B 642, 436 (2006); B.-A. Li, L.-W. Chen, C.M. Ko, A.W. Steiner, nuclth/06011028. 